\title{
Virulence Traits of Environmental and Clinical Legionella pneumophila Multilocus Variable-Number Tandem-Repeat Analysis (MLVA) Genotypes
}

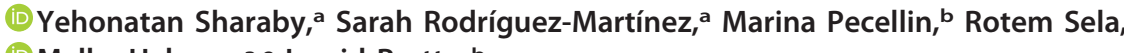 \\ (D) Malka Halpern, a,e Ingrid Brettar ${ }^{\mathrm{b}}$ \\ aDepartment of Evolutionary and Environmental Biology, Faculty of Natural Sciences, University of Haifa, Haifa, \\ Israel \\ bDepartment of Vaccinology and Applied Microbiology, Helmholtz Centre for Infection Research, \\ Braunschweig, Germany \\ ‘Clinical Microbiology Laboratory, Baruch Padeh Poriya Medical Center, Galilee, Tiberias, Israel \\ dThe Faculty of Medicine in the Galilee, Bar-llan University, Galilee, Tsfat, Israel \\ eDepartment of Biology and Environment, Faculty of Natural Sciences, University of Haifa, Oranim, Tivon, Israel
}

ABSTRACT Legionella pneumophila causes water-based infections resulting in severe pneumonia. Recently, we showed that different MLVA-8 (multilocus variablenumber tandem-repeat analysis using 8 loci) genotypes dominated different sites of a drinking-water distribution system. Each genotype displayed a unique temperaturedependent growth behavior. Here we compared the pathogenicity potentials of different MLVA-8 genotypes of environmental and clinical strains. The virulence traits studied were hemolytic activity and cytotoxicity toward amoebae and macrophages. Clinical strains were significantly more hemolytic than environmental strains, while their cytotoxicity toward amoebae was significantly lower at $30^{\circ} \mathrm{C}$. No significant differences were detected between clinical and environmental strains in cytotoxicity toward macrophages. Significant differences in virulence were observed between the environmental genotypes (Gt). Gt15 strains showed a significantly higher hemolytic activity. In contrast, Gt4 and Gt6 strains were more infective toward Acanthamoeba castellanii. Moreover, Gt4 strains exhibited increased cytotoxicity toward macrophages and demonstrated a broader temperature range of amoebal lysis than Gt6 and Gt15 strains. Understanding the virulence traits of Legionella genotypes may improve the assessment of public health risks of Legionella in drinking water.

IMPORTANCE Legionella pneumophila is the causative agent of a severe form of pneumonia. Here we demonstrated that clinical strains were significantly more cytotoxic toward red blood cells than environmental strains, while their cytotoxicity toward macrophages was similar. Genotype 4 (Gt4) strains were highly cytotoxic toward amoebae and macrophages and lysed amoebae in a broader temperature range than to the other studied genotypes. The results can explain the relatively high success of Gt4 in the environment and in clinical samples; thus, Gt4 strains should be considered a main factor for the assessment of public health risks of Legionella in drinking water. Our findings shed light on the ecology, virulence, and pathogenicity potential of different $L$. pneumophila genotypes, which can be a valuable parameter for future modeling and quantitative microbial risk assessment of Legionella in drinking-water systems.

KEYWORDS virulence, pathogenicity, hemolysis, cytotoxicity, amoeba, macrophages, Legionella pneumophila, MLVA-8 genotyping
Received 19 February 2018 Accepted 25 February 2018

Accepted manuscript posted online 9 March 2018

Citation Sharaby Y, Rodríguez-Martínez S, Pecellin M, Sela R, Peretz A, Höfle MG, Halpern

M, Brettar I. 2018. Virulence traits of environmental and clinical Legionella pneumophila multilocus variable-number tandem-repeat analysis (MLVA) genotypes. Appl Environ Microbiol 84:e00429-18. https://doi.org/10.1128/AEM .00429-18.

Editor Charles M. Dozois, INRS - Institut Armand-Frappier

Copyright $\odot 2018$ American Society for Microbiology. All Rights Reserved.

Address correspondence to Malka Halpern, mhalpern@research.haifa.ac.il. 
egionella pneumophila, the causative agent of legionellosis, is a relevant bacterial pathogen in developed countries (1-3). Members of the genus Legionella inhabit both natural and man-made freshwater environments around the globe $(1,4-6)$. The transfer of $L$. pneumophila from these environmental reservoirs to humans via inhalation of contaminated water aerosols is considered the major source of infection $(5,7)$. The abundance of man-made freshwater reservoirs and the resulting increase in distribution of their water via aerosols has led to increased human exposure to Legionella and increased infection rates compared to those in past decades (7). Since 2013, about 5,000 sporadic cases of legionellosis and at least 20 outbreaks were reported annually to the Centers for Disease Control and Prevention in the United States (8). In contrast, only one case of person-to-person transmission has been documented; therefore, human infection is considered a dead end for L. pneumophila transmission $(9,10)$.

Rowbotham (11) described the ability of L. pneumophila to multiply intracellularly within freshwater protozoa. Since then, a broad set of protozoan species have been described as hosts of this species $(12,13)$. Understanding this host-parasite interaction from protozoa to macrophages is important for understanding both the pathogenesis and the ecology of $L$. pneumophila $(14,15)$. Upon reaching the lungs, $L$. pneumophila infects and proliferates within the immune system's macrophages, destroying them in the process (16). In addition, the pathology of Legionella infections in humans also involves alveolar cell destruction, and several L. pneumophila effector proteins and mechanisms are known to contribute to this ability (17-22). The evolution of virulence traits in L. pneumophila is suggested to result mainly from the microorganism's need to replicate in a host cell, reprogramming the host cell in favor of its needs for replication and thereby avoiding predation (23-25). The interaction of L. pneumophila with eukaryotic cells (protozoans or macrophages) is therefore a key to understanding the ability of the pathogen to cause disease (26). Many of the factors that promote its survival and replication in amoebae are also required for infection and growth in human macrophages $(24,27-29)$. It has been suggested that $L$. pneumophila possesses strain-specific virulence traits $(13,30,31)$. One important virulence factor, of relevance for protozoa and human cells, is the Dot/lcm type IVB translocation system. Currently, it has been shown that the Dot/Icm type IVB system is responsible in the strain $L$. pneumophila Philadelphia-1 for the translocation of over 330 proteins into the host cell, which represents over $10 \%$ of the proteome of this strain (32). Most of these effectors have been shown to have functional redundancy $(20,25,29,33-36)$; therefore, the lack of some of them does not usually decrease or eliminate the virulence of the strain (16, 26,32 ). So far, it is not completely understood what makes certain strains more pathogenic than others.

Since L. pneumophila reaches the human lungs via water aerosols, a thorough understanding of its ecology outside the human body and especially in drinking-water supply systems is important to enable design of efficient prevention measures (13, 37-40). From an epidemiological point of view, genotyping is important for determining the sources and the transfer pathway of infections. Moreover, differences in ecological traits and pathogenicity potential were found for different $L$. pneumophila genotypes colonizing drinking-water distribution systems (DWDSs) (38, 41-45). Multilocus variable-number tandem-repeat analysis using 8 loci (MLVA-8) is a genotyping method based on the variability found in eight tandemly repeated DNA sequences that represent sources of genetic polymorphism (minisatellites) $(45,46)$. Recent work by our group has shown that different sites of the same water network were dominated by different MLVA-8 genotypes (43). Analysis of the three dominant genotypes, genotype 4 (Gt4), Gt6, and Gt15, showed that these genotypes had a distinct range for temperature and abundance at their site of dominance. Therefore, these genotypes can be addressed as different ecotypes, meaning that each of them is adapted to a specific environmental condition. Gt4 and Gt6 strains belong to serogroup 1 and correspond to sequence type 1 (ST1) by sequence base typing, whereas Gt15 strains belong to serogroup 3 (for more details, see Table 6 and Fig. 7 in reference 43). Recently, Sharaby 
TABLE 1 Legionella pneumophila genotypes used in the current study ${ }^{a}$

\begin{tabular}{|c|c|c|c|c|c|c|c|}
\hline \multirow[b]{2}{*}{ Strain type } & \multirow[b]{2}{*}{$\begin{array}{l}\text { Sampling } \\
\text { point(s) }\end{array}$} & \multirow[b]{2}{*}{$\begin{array}{l}\text { MLVA-8 } \\
\text { genotype }(n)\end{array}$} & \multirow[b]{2}{*}{$\begin{array}{l}\text { Sequence } \\
\text { type, } \\
\text { serogroup }^{b}\end{array}$} & \multicolumn{4}{|c|}{$\begin{array}{l}\text { Temp }\left({ }^{\circ} \mathrm{C}\right) \text { for optimal value of } \\
\text { growth parameter: }\end{array}$} \\
\hline & & & & $\begin{array}{l}\text { Lag } \\
\text { phase } \\
(\lambda)^{c}\end{array}$ & $\begin{array}{l}\text { Maximum } \\
\text { growth } \\
\text { rate }\left(\mu_{\mathrm{m}}\right)^{c}\end{array}$ & $\begin{array}{l}\text { Maximum } \\
\text { density } \\
(A)^{c}\end{array}$ & $\begin{array}{l}\text { Growth } \\
\text { range }\end{array}$ \\
\hline \multirow[t]{3}{*}{ Environmental $^{d}$} & A & Gt15 (6) & NA, 3 & 25 & 42 & 37 & $<25-45$ \\
\hline & $C, D$ & Gt4 (19) & ST1, 1 & 25 & 42 & 25 & $<25-42$ \\
\hline & $E, F, G$ & Gt6 (13) & ST1, 1 & 25 & 37 & 35 & $<25-42$ \\
\hline \multirow[t]{6}{*}{ Clinical } & Hospital & Gt4 (4) & ST1, 1 & 25 & 37 & 37 & $<25-42$ \\
\hline & Hospital & Gt6 (2) & $\mathrm{ST} 1,1$ & 25 & 37 & 39 & $<25-42$ \\
\hline & Hospital & Gt19 (1) & ST1, 1 & 25 & 37 & 37 & $<25-42$ \\
\hline & Hospital & Gt20 (1) & $\mathrm{ST} 1,1$ & 25 & 37 & 37 & $<25-42$ \\
\hline & Hospital & Gt22 (2) & ST59, 1 & 25 & 37 & 42 & $<25-42$ \\
\hline & Hospital & Gt24 (2) & ST93, 1 & 25 & 37 & 37 & $<25-42$ \\
\hline
\end{tabular}

aFor more details, see reference 37 .

bSequence types are according to sequence-base typing. NA, not available.

cAverage of the optimal temperature for the studied strains.

The indicated sampling points in the drinking-water network were representative of the whole network; flow direction was from sampling point $A$ to point $G$. Additional environmental strains were used for

hemolytic activity assessment. Details regarding these isolates can also be found in Table S3 in reference 43.

et al. (37) demonstrated that the distribution and temperature preferences of these environmental genotypes were consistent with their temperature-dependent growth traits. For clinical isolates, it was shown that they had significantly higher growth rates and reached higher maximal cell densities at $37^{\circ} \mathrm{C}$ and $42{ }^{\circ} \mathrm{C}$ than the environmental strains (37). These findings highlight the importance of studying and characterizing the physiological and virulence traits of different L. pneumophila genotypes in order to promote our understanding of the hazardous potential of pathogenic strains.

Here we analyzed and compared virulence traits of L. pneumophila MLVA-8 genotypes isolated from drinking water as well as from clinical sources. We characterized the bacterial contact-dependent hemolytic activity toward red blood cells (RBC) and their cytotoxicity toward macrophages and amoebae. Hemolytic activity was assessed as an indicator of pore-forming activity $(17,47)$. The results demonstrated significant differences in the virulence traits between the different genotypes. Furthermore, clinical strains exhibited significantly higher pore-forming activity toward RBC than environmental strains. Our findings demonstrate that it is important to identify L. pneumophila genotypes in order to assess public health risks of Legionella in DWDSs.

\section{RESULTS}

A total of 61 environmental and 12 clinical L. pneumophila strains were studied. The environmental strains represent a subset of strains belonging to three genotypes that dominated a water network in northern Israel and belonged to MLVA-8 genotypes 4, 6 , and 15 (43) (Table 1). Six of the clinical strains belonged to Gt4 and Gt6, and the rest represented a diverse set of genotypes (Gt19, Gt20, Gt22, and Gt24) (37) (Table 1).

Toxicity toward amoebae. Amoeba infection was studied using 20 environmental and 12 clinical L. pneumophila strains (Table 1). The survival of amoebae after 12 and $24 \mathrm{~h}$ of exposure to L. pneumophila strains was compared to that of amoebae without Legionella infection (control). The results are summarized in Fig. 1 as the average percentage of Acanthamoeba castellanii survival after exposure to multiple strains of the same isolation source (Fig. 1A) and MLVA-8 genotypes (Fig. 1B). Detailed data for individual strains are provided in Table S1 in the supplemental material.

(i) Clinical versus environmental strains. No significant differences were observed between the survival rates of amoebae after exposure to $L$. pneumophila strains from clinical and environmental sources at $25^{\circ} \mathrm{C}$ (after $12 \mathrm{~h}$ and $24 \mathrm{~h}$ of infection). At $30^{\circ} \mathrm{C}$, amoeba survival rates were significantly higher after a 24-h infection by the clinical strains than after such an infection with the environmental strains (Student's $t$ test [one 

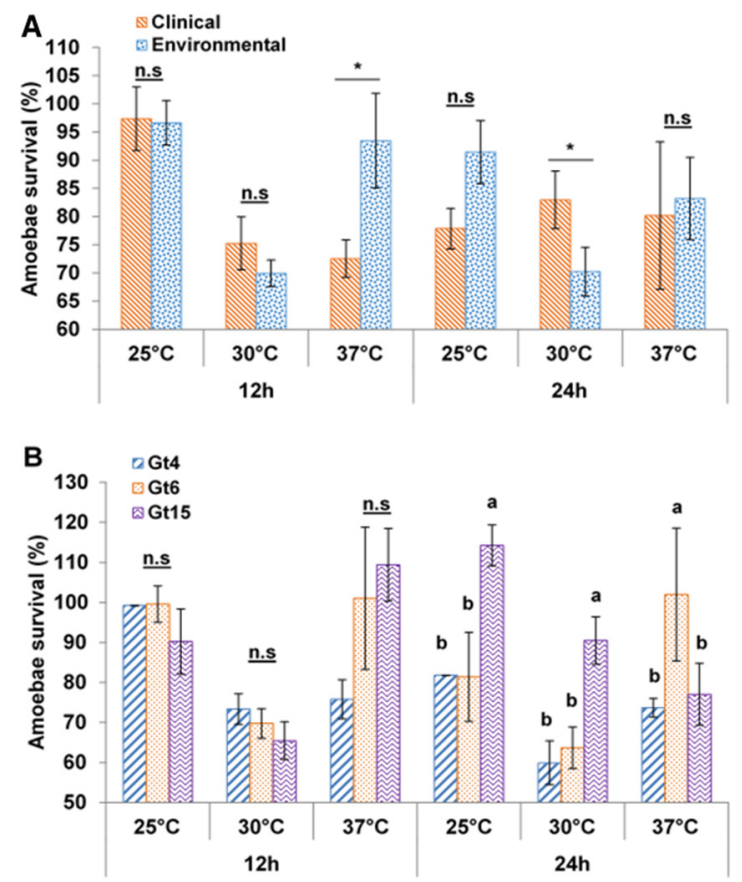

FIG 1 Percentages of live $A$. castellanii cells after infection with L. pneumophila strains (mean \pm standard error of intact amoeba cells relative to the control) at different temperatures $\left(25^{\circ} \mathrm{C}, 30^{\circ} \mathrm{C}\right.$, and $37^{\circ} \mathrm{C}$ ) and exposure durations (12 and 24 h). (A) Comparison between environmental $(n=20)$ and clinical $(n=12)$ strains. (B) Comparison between different environmental L. pneumophila genotypes (Gt4 $[n=8]$, Gt6 $[n=6]$, and Gt15 $[n=6]$ ). Different letters above the bars indicate significant differences between genotypes tested with one-way ANOVA and Tukey's honestly significant difference (HSD) post hoc test with a confidence interval of 95\%; asterisks indicate significant differences between clinical and environmental strains according to Student's $t$ test. Note that in some cases the amoeba cells replicated during the assay so that at the end of the assay there were more amoeba cells than at the beginning of the assay. More details can be found in Table S1 in the supplemental material. n.s., not significant.

tail], $t_{30}=1.86$ and $\left.P=0.037\right)$. In contrast, amoeba survival rates were significantly higher after $12 \mathrm{~h}$ of infection by the environmental strains than after that with the clinical strains at $37^{\circ} \mathrm{C}$ (Student's $t$ test, $t_{27 \cdot 04}=2.86$ and $P=0.008$ ). Thus, clinical strains exhibited a superior cytotoxicity potential toward amoebae at $37^{\circ} \mathrm{C}$ after a $12-\mathrm{h}$ exposure. However, no significant differences in amoeba survival rates between the environmental and the clinical strains were detected after a $24-\mathrm{h}$ exposure at $37^{\circ} \mathrm{C}$ (Fig. 1A).

(ii) Environmental genotypes. Amoeba survival rates were not significantly different at $25^{\circ} \mathrm{C}, 30^{\circ} \mathrm{C}$, and $37^{\circ} \mathrm{C}$ after $12 \mathrm{~h}$ of incubation with L. pneumophila (Fig. 1B). However, amoeba survival rates varied significantly after $24 \mathrm{~h}$ of incubation with different $L$. pneumophila genotypes at $25^{\circ} \mathrm{C}, 30^{\circ} \mathrm{C}$, and $37^{\circ} \mathrm{C}$ (one-way analysis of variance [ANOVA]: $F_{2,17}=5.16$ and $P=0.018, F_{2,17}=8.58$ and $P=0.003$, and $F_{2,17}=$ 4.64 and $P=0.026$, respectively). After a 24-hour incubation period at the lower temperatures of $25^{\circ} \mathrm{C}$ and $30^{\circ} \mathrm{C}$, amoeba survival rates were similar when exposed to Gt4 and Gt6 strains and significantly higher when exposed to Gt15 strains (Fig. 1B). In contrast, amoeba survival rates were significantly lower after a 24-hour exposure to Gt4 and Gt15 strains than after exposure to Gt6 strains at $37^{\circ} \mathrm{C}$ (Fig. 1B). Gt4 strains were able to significantly reduce amoeba survival rates at all three studied temperatures, with $28 \%$ average reduction after 12- and 24-hour exposure periods. Gt6 strains reduced amoeba survival rates only at the lower temperatures, whereas at $37^{\circ} \mathrm{C}$ an increase in amoeba concentrations was observed compared to the control (amoebae without Legionella). The reverse response to temperature was observed after amoebae were exposed to Gt15 strains. A significant increase in amoeba concentrations was observed compared to those of the control at the lower temperatures, whereas at $37^{\circ} \mathrm{C}$ 

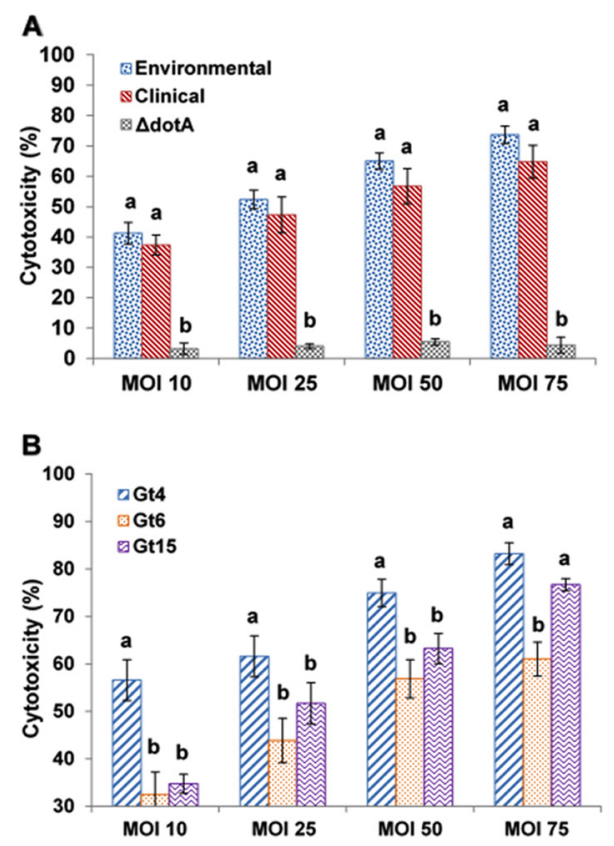

FIG 2 L. pneumophila cytotoxicity toward THP-1 macrophage-like cells. (A) Comparison between environmental $(n=15)$ and clinical $(n=12)$ strains and the $\Delta \operatorname{dotA}$ mutant strain (L. pneumophila Philadelphia-1 icm/dot-deficient mutant strain). (B) Comparison between environmental strains of different MLVA-8 genotypes ( $n=5$ for each genotype). The percentage of the strain's cytotoxicity was calculated as the reduction in THP-1 cell viability (mean \pm standard error) relative to the control. Multiplicity of infection (MOI) refers to the ratio between L. pneumophila cells and THP-1 monocytes (e.g., an $\mathrm{MOI}$ of 10 indicates a 10:1 ratio of L. pneumophila cells to THP-1 cells). Different letters above the bars indicate significant differences between genotypes by one-way ANOVA and Tukey's HSD post hoc test with a confidence interval of $95 \%$.

Gt15 strains significantly reduced amoeba survival rates compared to those of Gt6 strains (Fig. 1B).

The ability of L. pneumophila strains to decrease amoeba concentrations after 12 and $24 \mathrm{~h}$ of coculture was highly variable for each genotype and was correlated with the strains' temperature-dependent growth kinetics (the test was performed using yeast extract broth [37]). The highest suppression of amoeba proliferation was observed for Gt4 environmental strains. This was observed at all studied temperatures.

Environmental Gt4 strains reduced amoeba concentrations at the different temperatures to lower levels than the clinical strains and also than the other environmental genotypes (Gt6 and Gt15) that had a restricted temperature range (Fig. 1B). However, the comparison of clinical versus environmental Gt4 strains showed no significant differences in cytotoxicity toward $A$. castellanii at the three studied temperatures $\left(25^{\circ} \mathrm{C}\right.$, $t_{10}=1.01 ; 30^{\circ} \mathrm{C}, t_{10}=1.33$; and $37^{\circ} \mathrm{C}, t_{10}=1.85[P>0.05]$ ) (cytotoxicity results for $\mathrm{Gt} 4$ clinical and environmental strains can be found in Table S1).

Toxicity toward macrophages. The cytotoxicities of L. pneumophila strains toward macrophages were studied and compared using a randomly chosen subset of 15 environmental isolates (five of each of the genotypes Gt4, Gt6, and Gt15) and seven clinical isolates (Table 1). The macrophage infection assays were performed at $37^{\circ} \mathrm{C}$ with four multiplicities of infection (MOIs) $(10,25,50$, and 75). The MOI is the ratio between the bacterial and the macrophage numbers. Results for each of the studied strains are summarized in Fig. 2 as the average cytotoxicity percentage after exposure to multiple strains from the same isolation source (Fig. 2A) and MLVA-8 genotype (Fig. 2B). Detailed data for individual strains are provided in Table S1.

Clinical strains reduced macrophage viability between $37.3 \% \pm 3.3 \%$ and $64.8 \% \pm$ $5.4 \%$ on average (MOI, between 25 and 75, respectively). No significant differences in cytotoxicity were detected between the clinical and the environmental strains (Fig. 2A). 

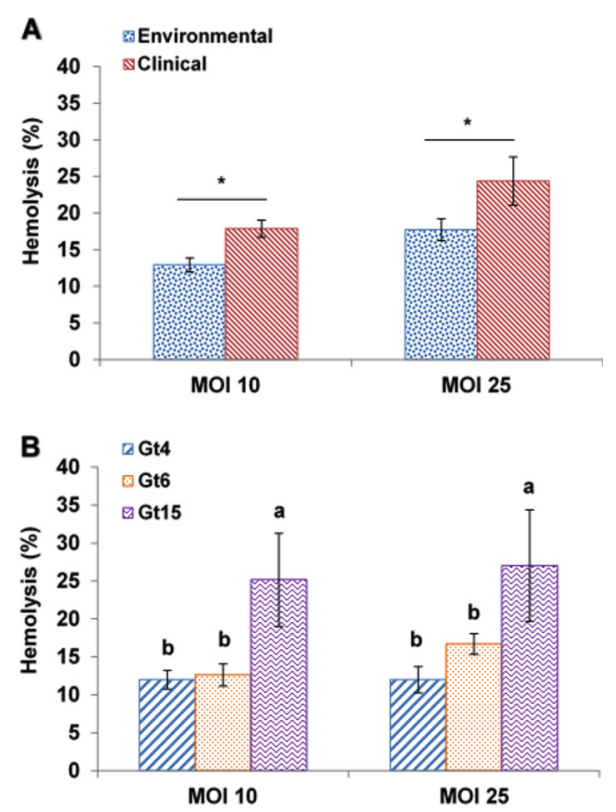

FIG 3 Hemolytic activity of L. pneumophila clinical and environmental strains against red blood cells (RBC). (A) Comparison of hemolytic activity toward RBC between environmental $(n=61)$ and clinical $(n=12)$ strains. (B) Comparison of hemolytic activity toward RBC between environmental MLVA-8 genotypes (Gt4, 19 strains; Gt6, 13 strains; Gt15, 6 strains). The hemolysis percentage was calculated compared to the positive control and was measured by $\mathrm{OD}_{415}$ (mean \pm standard error). Multiplicity of infection (MOI) refers to the ratio between L. pneumophila cells and RBC (e.g., an $\mathrm{MOI}$ of 10 indicates a 10:1 ratio of $L$. pneumophila cells to $\mathrm{RBC}$ ). Different letters above the bars indicate significant differences between groups tested with one-way ANOVA and Tukey's HSD post hoc test with a confidence interval of $95 \%$. Asterisks indicate significant differences between clinical and environmental strains according to Student's $t$ test.

As expected, the $\triangle$ dotA mutant strain exhibited significantly lower cytotoxicity toward macrophages $(48,49)$ than the environmental and the clinical isolates at all MOls $(3.1 \% \pm 1.9 \%$ to $4.4 \% \pm 2.6 \%$ reduction in macrophage viability at MOls of 25 to 75 , respectively) (Fig. 2A).

An average reduction of $41.3 \% \pm 3.6 \%$ to $73.7 \% \pm 2.9 \%$ in the macrophage viability was observed after their exposure to environmental strains with MOls between 25 and 75. Cytotoxicity rates were significantly higher after macrophages were exposed to Gt4 strains ( 56.6 to $83.2 \%$ reduction in THP-1 viability) than after exposure to Gt6 and Gt15 strains. These results were consistent at all four studied MOls (analysis of similarities [ANOSIM], $R=0.53$ and $P=0.002$ ). Cytotoxicity was the lowest after exposure to Gt6 strains (Fig. 2B). The comparison of clinical versus environmental Gt4 strains revealed significant differences in cytotoxicity at all four studied MOls. Environmental Gt4 strains were significantly more cytotoxic toward THP-1 macrophages than Gt4 strains of clinical origin ( $\mathrm{MOI}$ of $10, t_{6}=3.3$ and $P=0.016$; $\mathrm{MOI}$ of $25, t_{6}=3.6$ and $P=0.011$; $\mathrm{MOI}$ of $50, t_{6}=6.4$ and $P=0.001$; and $\mathrm{MOI}$ of $75, t_{6}=8.0$ and $P<0.001$ ).

Contact-mediated hemolysis. To study the ability of L. pneumophila to cause contact-mediated hemolysis, all isolates that were used for the amoeba and macrophage cytotoxicity assays (Table 1 ) and additional strains isolated from the same sampling campaign (43) were used. A total of 61 environmental strains (Gt4, $n=19$; Gt6, $n=13 ; \mathrm{Gt15}, n=6$; and isolates without genotype identities, $n=23$ ) and the 12 clinical strains were tested. The results are shown in Fig. 3 and Table S1 as percentages of hemolysis at $37^{\circ} \mathrm{C}$ (e.g., ability to create pores in red blood cell membranes compared to the control). Figure $3 \mathrm{~A}$ shows the average percentage of hemolysis after exposure to L. pneumophila strains isolated from clinical and environmental sources. Figure 3B summarizes the average percentage of hemolysis after red blood cells were exposed to environmental strains belonging to different MLVA-8 genotypes. Data for the individual strains are provided in Table S1. 


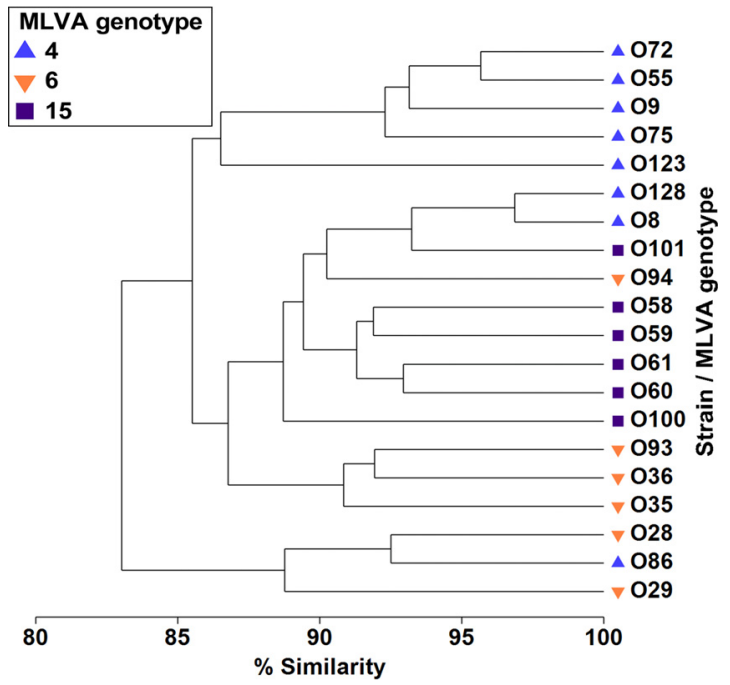

FIG 4 Agglomerative clustering dendrogram representing the percentage of similarity between virulence characteristics of $L$. pneumophila strains belonging to different genotypes (Gt4, Gt6, and Gt15). The resemblance matrix was calculated using the Bray-Curtis index of association on the following variables: (i) amoeba survival after exposure to L. pneumophila at different temperatures $\left(25^{\circ} \mathrm{C}, 30^{\circ} \mathrm{C}\right.$, and $\left.37^{\circ} \mathrm{C}\right)$ and durations (12 and 24 h), (ii) THP-1 cell viability after exposure to L. pneumophila at four MOls (10 to 75), and (iii) hemolytic ability at two MOls (10 and 25 ).

Clinical strains were significantly more hemolytic than ST1 strains isolated from environmental sources (e.g., environmental Gt4 and Gt6 strains). Although modest, these differences were observed at MOls of both $10\left(t_{52}=2.022, P<0.05\right)$ and 25 $\left(t_{52}=2.01, P<0.05\right)$ (Fig. 3A). No significant differences in hemolytic activity were observed when environmental strains originating from water and biofilm were compared $\left(t_{38}=0.98, P>0.05\right)$. In contrast, one-way ANOVA revealed significant differences in hemolytic activity between environmental strains from different genotypes (Fig. 3B). Gt15 strains were significantly more hemolytic than environmental Gt4 and Gt6 strains (one-way ANOVA, $F_{2,37}=3.7$ and $P=0.034$ ). In addition, the majority of Gt15 strains were more hemolytic than clinical strains at both MOls (Table S1). No significant differences were observed between clinical and environmental Gt 4 strains at MOls of both $10\left(t_{10}=1.56, P=0.15\right)$ and $25\left(t_{25}=1.32, P=0.22\right)$.

Summary of virulence characteristics. In summary, clinical strains seemed to be significantly more cytotoxic toward amoebae at $37^{\circ} \mathrm{C}$ (after a 12-hour infection) and significantly more hemolytic than environmental Gt4 and Gt6 strains. On the other hand, no significant differences were observed between clinical and environmental strains regarding their cytotoxicity toward macrophages (Fig. 2A).

Considering all the data, significant differences were detected between the virulence characteristics of environmental strains belonging to different genotypes (ANOSIM, $R=0.48$ and $P=0.001$ ). Gt4 strains were significantly more cytotoxic toward amoebae and macrophages than Gt6 and Gt15 strains (Fig. 1B and 2B). Furthermore, each genotype presented a unique virulence profile with respect to cytotoxicity toward amoebae and THP-1 monocytes as well as hemolytic activity (pairwise comparisons, $P<0.01$ ). Hierarchical agglomerative cluster analysis showed that strains belonging to the same genotype clustered together and formed three almost-homogenous clusters of strains with a genotype-dependent virulence profile (Fig. 4).

\section{DISCUSSION}

L. pneumophila is an opportunistic bacterial pathogen that besides parasitizing its natural amoeba hosts is also capable of infecting human cells. It does so by using a large set of effector proteins that have been acquired along its evolution with its natural amoeba hosts $(32,50)$. A recent study has compared the genomes of 38 Legionella species and has identified in total nearly 6,000 potential effector proteins 
(51). Elucidating the roles that the effectors play in the infection is usually complicated due to the high redundancy of effector functions $(30,32)$. Next to highly sophisticated proteomic analysis, the in vitro infection of host cells is a very useful experimental approach to assess the virulence phenotypes of $L$. pneumophila strains. Understanding L. pneumophila pathogenicity has been achieved mostly by the study of clinical isolates. Nevertheless, the analysis of the pathogenicities of environmental isolates is essential, since environmental strains are considered the primary source of outbreaks as well as nosocomial and community-acquired Legionnaires' disease (4, 13, 14, 52, 53).

In the current study, an extensive data set of genotyped isolates was used to determine potential differences between environmental and clinical isolates in the interaction with their primary model host systems. Twelve clinical and 61 environmental strains were used for amoeba and red blood cell assays. In addition, a subset of the strains (15 environmental and seven clinical strains) was used to study and compare their cytotoxicities toward macrophages. Generally, the degree of virulence of $L$. pneumophila strains is determined by studying various traits such as infectivity, intracellular replication, and cytotoxicity using macrophages. Moreover, specific strains are thought to be particularly pathogenic since they are frequently found among clinical isolates, although it is still not clear which specific factors promote their pathogenicity $(23,30,31)$.

The THP-1 human macrophage-like monocyte cell line and A. castellanii were selected for the cytotoxicity assays due to their extensive use as models for Legionella virulence $(18,29,30,54,55)$. Pore formation is a mechanism that contributes to destruction of alveolar epithelial cells and macrophages; thus, the strains' ability to cause pore formation using RBC (hemolytic activity) was assessed (17, 21, 22, 47, 56, 57). Our results demonstrated that there were no significant differences in the cytotoxicity toward macrophages between the clinical and environmental L. pneumophila strains (Fig. 1A). However, clinical strains exhibited a significantly higher hemolytic activity toward RBC than environmental strains (Fig. 2A). Regarding the MLVA genotypes, Gt4 strains were significantly more cytotoxic toward amoebae and macrophages (Fig. 1B and 2 B), while Gt15 strains had a higher hemolytic activity (Fig. 3B).

Mercante and Winchell (42) and McDade (58) recommended that pathogenicity and genotype relevance for public health should be assessed by isolate-based studies in macrophage and amoeba cytotoxicity assays. The resolution of MLVA genotyping applied in this study for L. pneumophila strains allowed the classification and comparison of the pathogenicity potential of each genotype in order to determine which genotype poses the greatest risk to public health. MLVA is especially useful because it allows a good resolution within the highly health-relevant and abundant sequence type 1 (ST1), comprising the reference strain L. pneumophila Paris. Our findings suggest that a high variability exists even among ST1 strains in terms of virulence and pathogenicity. Strains Gt4 and Gt6, both of which were studied here, belong to ST1. Gt4 strains were significantly more cytotoxic toward amoebae and macrophages, and as such, the presence of this genotype in DWDSs may pose a much more severe health risk than that of ST1 strains belonging to Gt6. However, the ability of L. pneumophila to cause illness in humans is dependent on its ability to multiply intracellularly within macrophages $(4,5,32,50)$. Thus, in order to have a more definitive answer regarding the abilities of the different genotypes to cause illness in humans, further research is needed, and MLVA genotypes belonging to ST1 strains should be compared with regard to their ability to proliferate inside macrophages.

With respect to ecophysiological and virulence traits, clinical and environmental strains differed and showed genotype-dependent characteristics, as shown in a recent study (37) and the current study. Sharaby et al. (37) demonstrated that (i) L. pneumophila strains (the same isolates as in the current study) showed genotype-specific temperature-dependent growth traits and (ii) clinical and environmental strains possessed significantly different temperature-dependent growth traits. In the current study, clinical strains were significantly more cytotoxic toward amoebae and more hemolytic than environmental strains (Fig. 1A and 3A; see Table S1 in the supplemental 
material). Significant differences were observed in the virulence of different genotypes, and each genotype presented a unique virulence profile (Fig. 4).

The interactions between L. pneumophila and the amoebae in DWDSs play an important role in its survival, replication, and stress tolerance $(25,59)$. Interestingly, most strains showed amoebal lysis from $25^{\circ} \mathrm{C}$ to $37^{\circ} \mathrm{C}$ irrespective of environmental or clinical origin. Exceptions were the high-temperature-adapted Gt15 strains that failed to lyse amoebae at $25^{\circ} \mathrm{C}$ and some strains of Gt6 that failed to lyse at $37^{\circ} \mathrm{C}$.

Gt4 showed the widest temperature range for amoebal lysis (i.e., from $25^{\circ} \mathrm{C}$ to $37^{\circ} \mathrm{C}$ ), the highest lysis activity, and the most long-lasting reduction of amoebal abundance. Gt4 was also able to reduce amoeba concentrations beyond $12 \mathrm{~h}$ at all temperatures. This was in contrast to the case for the other environmental or clinical genotypes (Fig. 1B).

Rodríguez-Martínez et al. (43), who isolated and identified the different genotypes that were examined in the current work, found that Gt4 strains were the most abundant in the studied drinking-water network (43). Moreover, L. pneumophila Paris is an example of a Gt4 strain (60). Strains closely related to L. pneumophila Paris were isolated from a broad climatic range from all over Europe, both from water distribution networks and from sporadic and outbreak-related legionellosis cases $(60,61)$. The results of the current study can explain the high abundance in the studied drinkingwater network (43) as well as the worldwide occurrence of strains closely related to $L$. pneumophila Paris (60) that also belong to Gt4. Gt4 strains have relatively high success in the environment, as they proliferate within the amoebae with a greater efficiency and in a broader temperature range than other genotypes. For example, the growth rates of Gt4 strains are superior to those of Gt15 and Gt6 strains at $25^{\circ} \mathrm{C}$ and $37^{\circ} \mathrm{C}$, respectively (37).

Conclusion. Our findings, together with those of Sharaby et al. (37), shed light on the ecology, virulence, and pathogenicity potential of different L. pneumophila MLVA-8 genotypes and may be used for health impact assessments of the risks posed by the presence of specific genotypes within drinking-water distribution systems. As far as we know, the current study showed for the first time a genotype-related temperature dependence of amoebal lysis for $L$. pneumophila strains. This aspect is of importance for the growth and survival of $L$. pneumophila genotypes in the environment and could be a valuable parameter for future modeling and quantitative microbial risk assessment. The current quantitative Legionella risk assessment models and public health impact assessments $(62,63)$ refer to all $L$. pneumophila serogroup 1 strains as the same in terms of health risks. The unique ecophysiological and virulence profiles of genotypes dominating a water network demonstrate the necessity for use of a genotype-related profile for public health management and risk assessment measures. Further study using comparative whole-genome analysis of these Gt4, Gt6, and Gt15 strains from both environmental and clinical sources will reveal the unique proliferation traits of Gt4 strains and may result in molecular tools for detecting this specific genotype in both water distribution systems and clinical specimens.

\section{MATERIALS AND METHODS}

L. pneumophila strains. The environmental strains in the current study comprised strains isolated from a small drinking-water system in northern Israel near Tivon city (coordinates: $32^{\circ} 42^{\prime} 43.17^{\prime \prime} \mathrm{N}$, $35^{\circ} 6^{\prime} 28.66^{\prime \prime} \mathrm{E}$ ). Briefly, one liter of water sample was filtered through a cellulose nitrate 0.2- $\mu \mathrm{m}$-pore-size filter (Sartorius Stedim Biotech, Germany). Two plates of the Legionella selective medium glycinevancomycin-polymyxin-cycloheximide (GVPC) medium (Becton Dickinson GmH, Germany) were plated with $0.5 \mathrm{ml}$ and $0.1 \mathrm{ml}$ of untreated water. One milliliter was thermally treated $\left(50^{\circ} \mathrm{C}\right.$ for $\left.30 \mathrm{~min}\right)$, and $0.5-\mathrm{ml}$ samples were plated on two GVPC plates. Sterile swabs were used for Legionella isolation from biofilm (for more details, see reference 43). All plates were incubated at $37^{\circ} \mathrm{C}$. After 7 days of incubation, five colonies of each positive sample were selected and isolated five more times. Isolates were kept in LB supplemented with $30 \%$ glycerol at $-80^{\circ} \mathrm{C}$. Genotyping was conducted using multilocus variablenumber tandem-repeat analysis using 8 loci (MLVA-8), and most of the strains belonged to three genotypes (Gt4, Gt6, and Gt15) (43). The clinical strains were isolated from sputum samples from pneumonia patients at Poriya and Rambam hospitals, northern Israel, and were described by Sharaby et al. (37). Table 1 describes the details of the environmental and clinical strains that were studied in the current work (isolation source, MLVA-8 genotype, serogroup, sequence type, and growth traits). The 
genotypes described in Table 1 were studied with respect to their amoeba and macrophage cytotoxicities and pore-forming capacity. For the hemolytic assay, a complete set of 61 environmental isolates retrieved in an environmental sampling campaign (43) were tested (details regarding these isolates can be found in Table S1 in the supplemental material and in Table S3 of reference 43). Twelve clinical strains from Israel were included in all assays.

Amoeba infection by $L$. pneumophila. Twenty environmental and 12 clinical strains (all described in Table 1) were used for studying the infection kinetics of strains with different L. pneumophila MLVA-8 genotypes toward amoebae. Amoeba infection assays were performed using A. castellanii (ATCC 50374) according to the protocol published by Rizzardi et al. (55). A. castellanii was cultured using proteose peptone-yeast-glucose (PYG) medium in accordance with ATCC protocols (medium 712) and with the addition of $50 \mathrm{ml}$ of $2 \mathrm{M}$ glucose (filter sterilized) (Sigma, USA). A. castellanii was incubated in PYG medium at 25 to $30^{\circ} \mathrm{C}$ for $72 \mathrm{~h}$. After incubation, cells were washed three times with sterile PYG, and then $5 \mathrm{ml}$ of PYG was added for the detachment of the amoebae from the flask surface. Amoebae were then counted in a Neubauer microscopy chamber (Bar-Naor, Israel), and cell concentrations were adjusted to a suspension of $1.25 \times 10^{4}$ cells $/ \mathrm{ml}$ in PYG. The amoeba culture was distributed into a 12 -well plate (Corning, USA) ( $2 \mathrm{ml}$ per well). Plates were centrifuged for $2 \mathrm{~min}$ at $500 \times g$ and then incubated for 3 $\mathrm{h}$ at $30^{\circ} \mathrm{C}$ in order for cells to adhere properly to the wells' surfaces. Each L. pneumophila isolate was inoculated in 15 to $20 \mathrm{ml}$ of yeast extract broth (YEB) with an initial optical density at $600 \mathrm{~nm}\left(\mathrm{OD}_{600}\right) \mathrm{Of}$ 0.1. The YEB medium $(2 \times)$ recipe was as follows: $10 \mathrm{~g}$ of ACES [ $N$-(2-acetamido)-2-aminoethanesulfonic acid] (Sigma, USA), $10 \mathrm{~g}$ of yeast extract (Conda, Spain), $1 \mathrm{~g}$ of ketoglutaric acid (Sigma, USA), $0.4 \mathrm{~g}$ of L-cysteine hydrochloride monohydrate (Sigma, USA), $0.25 \mathrm{~g}$ of iron(III) pyrophosphate (Sigma, USA), and $1,000 \mathrm{ml}$ of sterile distilled water. The medium was sterilized by filtration using a $0.22-\mu \mathrm{m}$-pore-size membrane (Corning, USA). After sterilization, the $\mathrm{pH}$ was adjusted to 6.9. The medium was diluted 1:1 with sterile distilled water before use. Bacterial cultures were incubated at $37^{\circ} \mathrm{C}$ until they reached the stationary phase of growth $(72 \mathrm{~h}$ ) and then were centrifuged at 2,500 $\times g$ for $15 \mathrm{~min}$. Supernatants were then discarded, and bacterial pellets were washed with phosphate-buffered saline (PBS) and resuspended in PYG medium to a final $\mathrm{OD}_{600}$ of 0.078 (corresponding to $6.25 \times 10^{7} \mathrm{CFU} / \mathrm{ml}$ ). The appropriate bacterial volume, which matched a 10:1 bacterium/amoeba ratio $(\mathrm{MOI}=10)$, was calculated and added to the 12-well plates. Plates were centrifuged for $5 \mathrm{~min}$ at $500 \times g$ to allow contact between amoebae and Legionella. Each L. pneumophila isolate was tested in five replicates. Positive controls (only amoebae in PYG medium) were also tested in five replicates. Negative controls (PYG medium) were used to ensure that no contaminations occurred. Plates were incubated at different temperatures $\left(25,30\right.$, or $\left.37^{\circ} \mathrm{C}\right)$ for 12 $\mathrm{h}$ and $24 \mathrm{~h}$ of infection. The plates were then placed on ice for $30 \mathrm{~min}$ for proper detachment of the amoebae from the bottom of the wells. Suspensions were mixed using a pipette, and intact amoebae were counted in Neubauer chambers.

Postinfection amoeba survival rates were calculated as the ratio of amoeba concentrations between the experimental wells and the average of the positive controls for each strain at each temperature. The percentage of amoeba survival was calculated using the following equation: percent amoeba survival = $A / B \times 100$, where $A$ is the amoeba concentration in the experiment (number of amoebae per milliliter) and $B$ is the average amoeba concentration in the uninfected positive controls (number of amoebae per milliliter). Earlier studies have shown that $A$. castellanii viability is not affected by the experimental conditions (e.g., temperature and incubation period) that were used in the current study $(64,65)$. Hence, the positive uninfected control (only A. castellanii in PYG medium) provided a solid estimate of the host proliferation rates and allowed the determination of L. pneumophila cytotoxicity toward A. castellanii.

Macrophage (THP-1) infection by L. pneumophila. A total of 27 L. pneumophila strains (15 environmental strains [5 each from the Gt4, Gt6, and Gt15 genotypes] and 12 clinical strains) (Table 1) were selected randomly to study macrophage (THP-1) infection by L. pneumophila. Before the infection experiments, L. pneumophila strains were grown in $50 \mathrm{ml}$ YEB medium that was diluted 1:2 with sterile water. Cultures were incubated at $37^{\circ} \mathrm{C}$ for $48 \mathrm{~h}$ to ensure that all strains had reached the stationary phase (the time needed for the strains to reach the stationary phase was determined previously). Bacterial density was assessed by $\mathrm{OD}_{600}$ (an $\mathrm{OD}$ of 0.7 was equivalent to $10^{9}$ bacteria/ml). Bacteria were diluted with RPMI 1640 modified medium (Gibco, Germany) to the specific multiplicity of infection (MOI). THP-1 monocyte cells (ATCC TIB-202TM) were maintained in RPMI 1640 modified medium (Gibco, Germany) containing 10\% fetal bovine serum (FBS) (Gibco, Germany) and 2 mM L-glutamine (Gibco, Germany) in a humid atmosphere containing $5 \% \mathrm{CO}_{2}$ at $37^{\circ} \mathrm{C}$. Before each infection experiment, exponentially growing THP-1 monocytes were washed with complete medium, counted, and incubated with phorbol-12-myristate-13-acetate (PMA) (Sigma-Aldrich, Germany) at a concentration of $100 \mathrm{ng} / \mathrm{ml}$ for $48 \mathrm{~h}$ in $5 \% \mathrm{CO}_{2}$ at $37^{\circ} \mathrm{C}$ to induce maturation of $10^{9}$ monocytes into macrophage-like adherent cells. Adherent cells were washed three times with PBS prior to infection. Cytotoxicity assays were carried out by infecting THP-1 monocytes seeded in 96-well plates at a density of $10^{5}$ cells per well with $L$. pneumophila strains at different MOls $(10,25,50$, and 75$)$ for $1 \mathrm{~h}$ at $37^{\circ} \mathrm{C}$ and $5 \% \mathrm{CO}_{2}$. To measure the percentage of viable monocytes after the infection, a colorimetric assay based on the oxidationreduction of resazurin acid (alamarBlue; Sigma-Aldrich, Germany) was used. After infection and washing with PBS, macrophages were incubated with $200 \mu \mathrm{l}$ of RPMI medium supplemented with $10 \%$ of alamarBlue. Absorbance was measured after $5 \mathrm{~h}$ at wavelengths of 570 and $600 \mathrm{~nm}$, using a Synergy 2 microplate reader (Biotek, Germany).

Cytotoxicity of $L$. pneumophila strains to the monolayers of macrophages was calculated as the ratio of the absorbance of the reduced alamarBlue of infected monolayers to that of the uninfected ones (66). Specifically, the percentage of cytotoxicity was calculated by the formula $\{[(\mathrm{O} 2 \times A 1)-(\mathrm{O} 1 \times \mathrm{A} 2)$ / $(\mathrm{O} 2 \times \mathrm{P} 1)-(\mathrm{O} 1 \times \mathrm{P} 2)] \times 100\}$, where $\mathrm{O} 1$ and $\mathrm{O} 2$ are the molar extinction coefficients of oxidized 
alamarBlue at 570 and $600 \mathrm{~nm}$, respectively, A1 and A2 are the absorbances of infected cells at 570 and $600 \mathrm{~nm}$, respectively, and P1 and P2 are the absorbances of noninfected cells at 570 and $600 \mathrm{~nm}$, respectively. The cytotoxicity of each $L$. pneumophila strain was measured in triplicates for each MOI. An $\mathrm{icm} /$ dot-deficient mutant $(\Delta \operatorname{dot} A$ ) of the reference strain L. pneumophila Philadelphia-1 (ATCC 33152) was used as a negative control.

Contact-mediated hemolysis assays. Quantification of the abilities of the clinical and environmental isolates to cause pore formation was achieved by assessing their hemolytic ability (i.e., their ability to create pores and lyse red blood cells). Contact-mediated hemolysis assays were performed for 61 environmental and 12 clinical isolates according to the procedure described by Flieger et al. (47). The tested strains included all isolates that were used for the amoeba and macrophage cytotoxicity assays and additional strains isolated in the same sampling campaign as described by Rodríguez-Martínez et al. (43) (details regarding the additional strains can be found in Table S3 in reference 43). Isolates were cultured on GVPC-buffered charcoal medium (BD, USA) for $72 \mathrm{~h}$. Sheep red blood cells (RBC) (Hylabs, Rehovot, Israel) were prepared for the assay. RBC (500 $\mu \mathrm{l})$ were diluted in $50 \mathrm{ml}$ of PBS (Hylabs, Rehovot, Israel). The cells were then washed three times by $10 \mathrm{~min}$ of centrifugation at $2,900 \times g$ until the supernatant was colorless. After washing, the RBC were resuspended in $40 \mathrm{ml}$ PBS and counted using a Neubauer microscopy chamber (Bar-Naor, Israel). The number of RBC in PBS was adjusted to concentrations of $1 \times 10^{8}$ and $2 \times 10^{8}$ cells $/ \mathrm{ml}$. Legionella isolates were suspended in $500 \mu \mathrm{l} \mathrm{PBS}$, and the optical density was measured at $\mathrm{OD}_{600}$ using a Synrergy HT microplate reader (BioTek, USA). Legionella cultures were then adjusted to fit the ratios of 1:10 (MOI, 10) and 1:25 (MOI, 25) RBC/bacterial cell count. Bacterial cells were then centrifuged for 2 min at maximum speed; $1 \mathrm{ml}$ of the RBC $\left(1 \times 10^{8}\right.$ cells $\left./ \mathrm{ml}\right)$ was added to the bacterial cells, mixed, and dispensed in 96-well plates with round bottoms (Greiner Bio-One, USA) (200 $\mu \mathrm{l}$ in each well, 5 replicates for each isolate at each concentration). Five wells of $200 \mu \mathrm{l} \mathrm{RBC} \mathrm{without}$ the addition of bacteria served as negative controls, and five wells of RBC with the addition of water served as positive controls $\left(100 \mu \mathrm{l}\right.$ of water plus $100 \mu \mathrm{l}$ of RBC $\left[1 \times 10^{8} \mathrm{cells} / \mathrm{ml}\right]$ ). Plates were then centrifuged at $3,120 \times g$ for $10 \mathrm{~min}$, followed by incubation at $37^{\circ} \mathrm{C}$ for $2 \mathrm{~h}$. After incubation, the pellet was resuspended and centrifuged again (at 3,120 $\times g$ for 2 to $5 \mathrm{~min}$ ). The supernatants were transferred to a 96-well flat-bottom plate (Corning, USA), and the $\mathrm{OD}_{415}$ was measured in order to determine the quantity of lysed RBC.

Statistical analysis. All statistical analysis was performed using IBM SPSS 20 and Primer7 software (Primer-e, Auckland, New Zealand). All tests were applied at a 95\% level of confidence. Student's $t$ test was applied to study the differences between the amoeba survival rates after 12 and $24 \mathrm{~h}$ of exposure to clinical $(n=12)$ versus environmental $(n=20)$ L. pneumophila strains at three different temperatures $\left(25^{\circ} \mathrm{C}, 30^{\circ} \mathrm{C}\right.$, and $\left.37^{\circ} \mathrm{C}\right)$. All groups were normally distributed according to the Shapiro-Wilk test $(P>0.05)$. Variances were equal between groups at all temperatures (Leven's test, $P>0.05$ ) except at $37^{\circ} \mathrm{C}$, and thus the Student $t$ test was conducted while not assuming equality of variances. One-way analysis of variance (ANOVA) was applied to study the differences between the amoeba survival rates after 12 and $24 \mathrm{~h}$ of exposure to the different L. pneumophila genotypes at three different temperatures $\left(25^{\circ} \mathrm{C}, 30^{\circ} \mathrm{C}\right.$, and $\left.37^{\circ} \mathrm{C}\right)$. All groups were normally distributed according to the Shapiro-Wilk test $(P>0.05)$. Variances were equal between groups at all temperatures (Leven's test, $P>0.05$ ) except at $37^{\circ} \mathrm{C}(12 \mathrm{~h}$ ). Analysis of similarities (ANOSIM) (67) was used to compare the cytotoxicity rates of the clinical and environmental L. pneumophila isolates as well as the different MLVA-8 genotypes toward A. castellanii. ANOSIM was applied on the following variables: percentage of amoeba survival relative to the control at different temperatures $\left(25^{\circ} \mathrm{C}, 30^{\circ} \mathrm{C}\right.$, and $37^{\circ} \mathrm{C}$ ) and infection durations (12 and $24 \mathrm{~h}$ ). An agglomerative clustering dendrogram (Fig. 4) was created using the Primer7 software in order to study the similarity between virulence characteristics of $L$. pneumophila strains belonging to different genotypes (Gt4, Gt6, and Gt15). The resemblance matrix was calculated using the Bray-Curtis index of association on the following variables: (i) amoeba survival after exposure to L. pneumophila at different temperatures $\left(25^{\circ} \mathrm{C}, 30^{\circ} \mathrm{C}\right.$, and $37^{\circ} \mathrm{C}$ ) and durations (12 and $24 \mathrm{~h}$ ), (ii) THP-1 cell viability after exposure to L. pneumophila at four MOls (10 to 75$)$, and (iii) hemolytic ability at two MOls (10 and 25$)$.

\section{SUPPLEMENTAL MATERIAL}

Supplemental material for this article may be found at https://doi.org/10.1128/AEM .00429-18.

SUPPLEMENTAL FILE 1, PDF file, $0.1 \mathrm{MB}$.

\section{ACKNOWLEDGMENTS}

This study was supported by a grant from the German Research Foundation (Deutsche Forschungsgemeinschaft [DFG]; grant GZ: HO 930/5-2) and by a grant from the United States-Israel Binational Science Foundation (BSF; grant 2015103).

We declare no conflicts of interest.

\section{REFERENCES}

1. Yu VL, Plouffe JF, Pastoris MC, Stout JE, Schousboe M, Widmer A, Summersgill J, File T, Heath CM, Paterson DL, Chereshsky A. 2002. Distribution of Legionella species and serogroups isolated by culture in patients with sporadic community-acquired legionellosis: an international collaborative survey. J Infect Dis 186:127-128. https://doi.org/10 $.1086 / 341087$. 
2. Cao B, Yao F, Liu X, Feng L, Wang L. 2013. Development of a DNA microarray method for detection and identification of all 15 distinct O-antigen forms of Legionella pneumophila. Appl Environ Microbiol 79:6647-6654. https://doi.org/10.1128/AEM.01957-13.

3. Marston BJ, Plouffe JF, File TM, Hackman BA, Salstrom S-J, Limpan HB, Kolxzak MS, Breiman RF. 1997. Incidence of community-acquired pneumonia requiring hospitalization. Results of a population-based active surveillance study in Ohio. Ann Intern Med 157:1709-1718.

4. Cunha BA, Burillo A, Bouza E. 2016. Legionnaires' disease. Lancet 387: 376-385. https://doi.org/10.1016/S0140-6736(15)60078-2.

5. Fields BS, Benson RF, Besser RE. 2002. Legionella and Legionnaires' disease: 25 years of investigation. Clin Microbiol Rev 15:506-526. https://doi.org/ 10.1128/CMR.15.3.506-526.2002.

6. Helbig JH, Bernander S, Castellani Pastoris M, Etienne J, Gaia V, Lauwers S, Lindsay D, Lück PC, Marques T, Mentula S, Peeters MF, Pelaz C, Struelens M, Uldum SA, Wewalka G, Harrison TG. 2002. Pan-European study on culture-proven Legionnaires' disease: distribution of Legionella pneumophila serogroups and monoclonal subgroups. Eur J Clin Microbiol Infect Dis 21:710-716. https://doi.org/10.1007/s10096-002-0820-3.

7. Abu Kwaik Y, Gao LY, Stone BJ, Venkataraman C, Harb OS. 1998. Invasion of protozoa by Legionella pneumophila and its role in bacterial ecology and pathogenesis. Appl Environ Microbiol 64:3127-3133.

8. Adams D, Fullerton K, Jajosky R, Sharp P, Onweh D, Schley A, Anderson W, Faulkner A, Kugeler K. 2015. Summary of notifiable infectious diseases and conditions-United States, 2013. MMWR Morb Mortal Wkly Rep 62:1-119. https://doi.org/10.15585/mmwr.mm6253a1.

9. Correia AM, Ferreira JS, Borges V, Nunes A, Gomes B, Capucho R, Gonçalves J, Antunes DM, Almeida S, Mendes A, Guerreiro M, Sampaio DA, Vieira L, Machado J, Simões MJ, Gonçalves P, Gomes JP. 2016. Probable person-to-person transmission of Legionnaires' disease. N Engl J Med 374:497-498. https://doi.org/10.1056/NEJMc1505356.

10. Borges V, Nunes A, Sampaio DA, Vieira L, Machado J, Simões MJ, Gonçalves P, Gomes JP. 2016. Legionella pneumophila strain associated with the first evidence of person-to-person transmission of Legionnaires' disease: a unique mosaic genetic backbone. Sci Rep 6:26261. https://doi .org/10.1038/srep26261.

11. Rowbotham TJ. 1980. Preliminary report on the pathogenicity of Legionella pneumophila for freshwater and soil amoebae. J Clin Pathol 33: 1179-1183. https://doi.org/10.1136/jcp.33.12.1179.

12. Fields BS. 1996. The molecular ecology of Legionellae. Trends Microbiol 4:286-290. https://doi.org/10.1016/0966-842X(96)10041-X.

13. Steinert M, Hentschel U, Hacker J. 2002. Legionella pneumophila: an aquatic microbe goes astray. FEMS Microbiol Rev 26:149-162. https:// doi.org/10.1111/j.1574-6976.2002.tb00607.x.

14. Swanson MS, Hammer BK. 2000. Legionella pneumophila pathogesesis: a fateful journey from amoebae to macrophages. Annu Rev Microbiol 54:567-613. https://doi.org/10.1146/annurev.micro.54.1.567.

15. Hoffmann C, Harrison CF, Hilbi H. 2014. The natural alternative: protozoa as cellular models for Legionella infection. Cell Microbiol 16:15-26. https://doi.org/10.1111/cmi.12235.

16. Steinert M, Heuner K, Buchrieser C, Albert-Weissenberger C, Glöckner G. 2007. Legionella pathogenicity: genome structure, regulatory networks and the host cell response. Int J Med Microbiol 297:577-587. https://doi .org/10.1016/j.ijmm.2007.03.009.

17. Alli OAT, Gao LY, Pedersen LL, Zink S, Radulic M, Doric M, Abu Kwaik Y. 2000. Temporal pore formation-mediated egress from macrophages and alveolar epithelial cells by Legionella pneumophila. Infect Immun 68: 6431-6440. https://doi.org/10.1128/IAI.68.11.6431-6440.2000.

18. Alli OAT, Zink S, von Lackum K, Abu-Kwaik Y. 2003. Comparative assessment of virulence traits in Legionella spp. Microbiology 149:631-641. https://doi.org/10.1099/mic.0.25980-0.

19. Banerji S, Aurass P, Flieger A. 2008. The manifold phospholipases A of Legionella pneumophila-identification, export, regulation, and their link to bacterial virulence. Int J Med Microbiol 298:169-181. https://doi.org/ 10.1016/j.jimm.2007.11.004.

20. Gomez-Valero L, Rusniok C, Cazalet C, Buchrieser C. 2011. Comparative and functional genomics of Legionella identified eukaryotic like proteins as key players in host-pathogen interactions. Front Microbiol 2:208. https://doi.org/10.3389/fmicb.2011.00208.

21. Kirby JE, Vogel JP, Andrews HL, Isberg RR. 1998. Evidence for poreforming ability by Legionella pneumophila. Mol Microbiol 27:323-336. https://doi.org/10.1046/j.1365-2958.1998.00680.x.

22. Shevchuk O, Jäger J, Steinert M. 2011. Virulence properties of the
Legionella pneumophila cell envelope. Front Microbiol 2:74. https://doi .org/10.3389/fmicb.2011.00074.

23. Molofsky AB, Swanson MS. 2004. Differentiate to thrive: lessons from the Legionella pneumophila life cycle. Mol Microbiol 53:29-40. https://doi .org/10.1111/j.1365-2958.2004.04129.x.

24. Khodr A, Kay E, Gomez-Valero L, Ginevra C, Doublet P, Buchrieser C, Jarraud S. 2016. Molecular epidemiology, phylogeny and evolution of Legionella. Infect Genet Evol 43:108-122. https://doi.org/10.1016/j .meegid.2016.04.033.

25. Isberg RR, O'Connor T, Heidtman M. 2009. The Legionella pneumophila replication vacuole: making a cozy niche inside host cells. Nat Rev Microbiol 7:13-24. https://doi.org/10.1038/nrmicro1967.

26. Newton HJ, Ang DKY, van Driel IR, Hartland EL. 2010. Molecular pathogenesis of infections caused by Legionella pneumophila. Clin Microbiol Rev 23:274-298. https://doi.org/10.1128/CMR.00052-09.

27. Cianciotto NP, Fields BS. 1992. Legionella pneumophila mip gene potentiates intracellular infection of protozoa and human macrophages. Proc Natl Acad Sci U S A 89:5188-5191.

28. Segal G, Shuman HA. 1999. Legionella pneumophila utilizes the same genes to multiply within Acanthamoeba castellanii and human macrophages. Infect Immun 67:2117-2124.

29. Faucher SP, Mueller CA, Shuman HA. 2011. Legionella pneumophila transcriptome during intracellular multiplication in human macrophages. Front Microbiol 2:60. https://doi.org/10.3389/fmicb.2011.00060.

30. Gomez-Valero L, Rusniok C, Rolando M, Neou M, Dervins-Ravault D, Demirtas J, Rouy Z, Moore RJ, Chen H, Petty NK, Jarraud S, Etienne J, Steinert M, Heuner K, Gribaldo S, Médigue C, Glöckner G, Hartland EL, Buchrieser C. 2014. Comparative analyses of Legionella species identifies genetic features of strains causing Legionnaires' disease. Genome Biol 15:505. https://doi.org/10.1186/s13059-014-0505-0.

31. D'Auria G, Jiménez-Hernández N, Peris-Bondia F, Moya A, Latorre A. 2010. Legionella pneumophila pangenome reveals strain-specific virulence factors. BMC Genomics 11:181. https://doi.org/10.1186/1471-2164 $-11-181$.

32. Ensminger AW. 2016. Legionella pneumophila, armed to the hilt: justifying the largest arsenal of effectors in the bacterial world. Curr Opin Microbiol 29:74-80. https://doi.org/10.1016/j.mib.2015.11.002.

33. Cazalet $C$, Rusniok $C$, Brüggemann $H$, Zidane $N$, Magnier $A$, Ma L, Tichit M, Jarraud S, Bouchier C, Vandenesch F, Kunst F, Etienne J, Glaser P, Buchrieser C. 2004. Evidence in the Legionella pneumophila genome for exploitation of host cell functions and high genome plasticity. Nat Genet 36:1165-1173. https://doi.org/10.1038/ng1447.

34. Chien M, Morozova I, Shi S, Sheng H, Chen J, Gomez SM, Asamani G, Hill K, Nuara J, Feder M, Rineer J, Greenberg JJ, Steshenko V, Park SH, Zhao B, Teplitskaya E, Edwards JR, Pampou S, Georghiou A, Chou I-C, lannuccilli W, Ulz ME, Kim DH, Geringer-Sameth A, Goldsberry C, Morozov P, Fischer SG, Segal G, Qu X, Rzhetsky A, Zhang P, Cayanis E, De Jong PJ, Ju J, Kalachikov S, Shuman HA, Russo JJ. 2004. The genomic sequence of the accidental pathogen Legionella pneumophila. Science 305: 1966-1968. https://doi.org/10.1126/science.1099776.

35. Galka F, Wai SN, Kusch H, Engelmann S, Hecker M, Schmeck B, Hippenstiel S, Uhlin BE, Steinert M. 2008. Proteomic characterization of the whole secretome of Legionella pneumophila and functional analysis of outer membrane vesicles. Infect Immun 76:1825-1836. https://doi.org/ 10.1128/IAI.01396-07.

36. Ninio S, Roy CR. 2007. Effector proteins translocated by Legionella pneumophila: strength in numbers. Trends Microbiol 15:372-380. https:// doi.org/10.1016/j.tim.2007.06.006.

37. Sharaby Y, Rodríguez-Martínez S, Oks O, Pecellin M, Mizrahi H, Peretz A, Brettar I, Höfle MG, Halpern M. 2017. Temperature-dependent growth modeling of environmental and clinical Legionella pneumophila multilocus variable-number tandem-repeat analysis (MLVA) genotypes. Appl Environ Microbiol 83:e03295-16. https://doi.org/10.1128/AEM.03295-16.

38. Sobral D, Le Cann P, Gerard A, Jarraud S, Lebeau B, Loisy-Hamon F, Vergnaud G, Pourcel C. 2011. High-throughput typing method to identify a non-outbreak-involved Legionella pneumophila strain colonizing the entire water supply system in the town of Rennes, France. Appl Environ Microbiol 77:6899-6907. https://doi.org/10.1128/AEM .05556-11.

39. Luck PC, Ecker C, Reischl U, Linde H-J, Stempka R. 2007. Cultureindependent identification of the source of an infection by direct amplification and sequencing of Legionella pneumophila DNA from a clinical specimen. J Clin Microbiol 45:3143-3144. https://doi.org/10.1128/ JCM.00685-07. 
40. Lesnik R, Brettar I, Höfle MG. 2016. Legionella species diversity and dynamics from surface reservoir to tap water: from cold adaptation to thermophily. ISME J 10:1064-1080. https://doi.org/10.1038/ismej .2015.199.

41. Lück C, Fry NK, Helbig JH, Jarraud S, Harrison TG. 2013. Typing methods for legionella. Methods Mol Biol 954:119-148. https://doi.org/10.1007/ 978-1-62703-161-5_6.

42. Mercante JW, Winchell JM. 2015. Current and emerging Legionella diagnostics for laboratory and outbreak investigations. Clin Microbiol Rev 28:95-133. https://doi.org/10.1128/CMR.00029-14.

43. Rodríguez-Martínez S, Sharaby Y, Pecellín M, Brettar I, Höfle M, Halpern M. 2015. Spatial distribution of Legionella pneumophila MLVA-genotypes in a drinking water system. Water Res 77:119-132. https://doi.org/10 .1016/j.watres.2015.03.010.

44. Vergnaud G, Pourcel C. 2009. Multiple locus variable number of tandem repeats analysis. Methods Mol Biol 551:141-158. https://doi.org/10 .1007/978-1-60327-999-4_12.

45. Pourcel C, Visca P, Afshar B, D'Arezzo S, Vergnaud G, Fry NK. 2007. Identification of variable-number tandem-repeat (VNTR) sequences in Legionella pneumophila and development of an optimized multiplelocus VNTR analysis typing scheme. J Clin Microbiol 45:1190-1199. https://doi.org/10.1128/JCM.02078-06.

46. Pourcel C, Vidgop Y, Ramisse F, Vergnaud G, Tram C. 2003. Characterization of a tandem repeat polymorphism in Legionella pneumophila and its use for genotyping. J Clin Microbiol 41:1819-1826. https://doi.org/ 10.1128/JCM.41.5.1819-1826.2003.

47. Flieger A, Rydzewski K, Banerji S, Broich M, Heuner K. 2004. Cloning and characterization of the gene encoding the major cell-associated phospholipase A of Legionella pneumophila, plaB, exhibiting hemolytic activity. Infect Immun 72:2648-2658. https://doi.org/10.1128/IAI.72.5.2648 -2658.2004 .

48. Berger KH, Isberg RR. 1993. Two distinct defects in intracellular growth complemented by a single genetic locus in Legionella pneumophila. Mol Microbiol 7:7-19. https://doi.org/10.1111/j.1365-2958.1993.tb01092.x.

49. Roy CR, Berger $\mathrm{KH}$, Isberg RR. 1998. Legionella pneumophila DotA protein is required for early phagosome trafficking decisions that occur within minutes of bacterial uptake. Mol Microbiol 28:663-674. https:// doi.org/10.1046/j.1365-2958.1998.00841.x.

50. Albert-Weissenberger C, Cazalet C, Buchrieser C. 2007. Legionella pneumophila--a human pathogen that co-evolved with fresh water protozoa. Cell Mol Life Sci 64:432-448. https://doi.org/10.1007/s00018 $-006-6391-1$

51. Burstein D, Amaro F, Zusman T, Lifshitz Z, Cohen O, Gilbert JA, Pupko T, Shuman HA, Segal G. 2016. Genomic analysis of 38 Legionella species identifies large and diverse effector repertoires. Nat Genet 48:167-175. https://doi.org/10.1038/ng.3481.

52. Atlas RM. 1999. Legionella: from environmental habitats to disease pathology, detection and control. Environ Microbiol 1:283-293. https:// doi.org/10.1046/j.1462-2920.1999.00046.x.

53. Stout J, Yu V. 1997. Legionellosis. N Engl J Med 337:682-687. https:// doi.org/10.1056/NEJM199709043371006.
54. Buse HY, Brehm A, Santo Domingo JW, Ashbolt NJ. 2011. Screening-level assays for potentially human-infectious environmental Legionella spp. J Microbiol 49:200-207. https://doi.org/10.1007/s12275-011-0233-z.

55. Rizzardi K, Winiecka-Krusnell J, Ramliden M, Alm E, Andersson S, Byfors S. 2015. Legionella norrlandica sp. nov., isolated from the biopurification systems of wood processing plants. Int J Syst Evol Microbiol 65:598-603. https://doi.org/10.1099/ijs.0.068940-0.

56. Eisenreich W, Heuner K. 2016. The life stage-specific pathometabolism of Legionella pneumophila. FEBS Lett 590:3868-3886. https://doi.org/10 .1002/1873-3468.12326.

57. Zuckman DM, Hung JB, Roy CR. 1999. Pore-forming activity is not sufficient for Legionella pneumophila phagosome trafficking and intracellular growth. Mol Microbiol 32:990-1001. https://doi.org/10.1046/j .1365-2958.1999.01410.x.

58. McDade JE. 2008. Legionella and the prevention of legionellosis. Emerg Infect Dis 14:1006a-1006. https://doi.org/10.3201/eid1406.080345.

59. Harb OS, Gao L-Y, Kwaik YA. 2000. From protozoa to mammalian cells: a new paradigm in the life cycle of intracellular bacterial pathogens. Environ Microbiol 2:251-265. https://doi.org/10.1046/j.1462-2920.2000.00112.x.

60. Visca P, D'Arezzo S, Ramisse F, Gelfand Y, Benson G, Vergnaud G, Fry NK, Pourcel C. 2011. Investigation of the population structure of Legionella pneumophila by analysis of tandem repeat copy number and internal sequence variation. Microbiology 157:2582-2594. https://doi.org/10.1099/mic.0.047258-0.

61. Aurell H, Etienne J, Forey F, Reyrolle M, Girardo P, Farge P, Decludt B, Campese C, Vandenesch F, Jarraud S. 2003. Legionella pneumophila serogroup 1 strain Paris: endemic distribution throughout France. J Clin Microbiol 41:3320-3322. https://doi.org/10.1128/JCM.41.7.3320 $-3322.2003$.

62. Armstrong TW, Haas CN. 2008. Legionnaires' disease: evaluation of a quantitative microbial risk assessment model. J Water Health 6:149-166. https://doi.org/10.2166/wh.2008.026.

63. Azuma K, Uchiyama I, Okumura J. 2013. Assessing the risk of Legionnaires' disease: the inhalation exposure model and the estimated risk in residential bathrooms. Regul Toxicol Pharmacol 65:1-6. https://doi.org/ 10.1016/j.yrtph.2012.11.003.

64. Moffat JF, Tompkins LS. 1992. A quantitative model of intracellular growth of Legionella pneumophila in Acanthamoeba castellanii. Infect Immun 60:296-301.

65. Ohno A, Kato N, Sakamoto R, Kimura S, Yamaguchi K. 2008. Temperaturedependent parasitic relationship between Legionella pneumophila and a free-living amoeba (Acanthamoeba castellanii). Appl Environ Microbiol 74: 4585-4588. https://doi.org/10.1128/AEM.00083-08.

66. Kwaik YA, Gao L-Y, Harb OS, Stone BJ. 1997. Transcriptional regulation of the macrophage-induced gene (gspA) of Legionella pneumophila and phenotypic characterization of a null mutant. Mol Microbiol 24:629-642. https://doi.org/10.1046/j.1365-2958.1997.3661739.x.

67. Clarke KR. 1993. Non-parametric multivariate analyses of changes in community structure. Aust J Ecol 18:117-143. https://doi.org/10.1111/j .1442-9993.1993.tb00438.x. 